\title{
La enseñanza de las expresiones algebraicas en octavo grado
}

\author{
Bra. Jasmina del Carmen Mejía García, Bra. María de los Ángeles Villagra Jarquín, \\ Br. Humberto Ramón Rojas Murillo.
}

Facultad Regional Multidisciplinaria de Chontales. ${ }^{1}$

Recibido 24 de abril 2013- Aprobado 28 de octubre 2013.

\section{RESUMEN}

Uno de los problemas más frecuentes que manifiestan los estudiantes de octavo grado es el temor ante el inicio del contenido de Álgebra. Ellos expresan que les resultaba difícil comprender que con la unión de números y letras podrían dar solución a problemas matemáticos. Sin embargo, se daban cuenta que es parte de su aprendizaje conocer y dominar este contenido. Pero ¿cómo hacer esta transición fácil y hasta divertida para nuestros estudiantes? ¿Cómo plantear situaciones del contexto con expresiones Algebraicas? Estas y otras preguntas fueron las que motivaron e inspiraron a realizar una investigación en este tema en particular: "Las expresiones Algebraicas". El desarrollo de cada uno de los procesos de esta investigación reveló que si los docentes se lo proponían, si se les dotará de más recursos didácticos y si juntos (padres, maestros, técnicos, comunidad educativa, etc.) unían esfuerzos para mejorar la "Calidad de la educación"; se podría brindar al estudiante una clase atractiva y dinámica, con la cual todos se sintieran identificados. Este trabajo reúne una serie de tópicos de interés para el docente y alumno. Además de algunas técnicas y estrategias innovadoras que contribuirán a lograr un vínculo entre el alumno y las matemáticas, destacando en todo momento la importancia de la asignatura y sobre todo del Álgebra, que permite analizar, modelar y resolver problemas en las diferentes áreas del conocimiento humano y la interpretación de los mismos, para mejorar el futuro académico y relacionarlo con la realidad del mundo que lo rodea.

Palabras clave: álgebra, estrategias, metodología, octavo grado, matemáticas.

\section{INTRODUCCIÓN}

Así como la aritmética surgió de la necesidad que tenían los pueblos primitivos de medir el tiempo y de contar sus posesiones, el origen del álgebra es muy posterior puesto que debieron de transcurrir muchos siglos para que le hombre llegara al concepto abstracto de número. Como parte de las matemáticas el álgebra tiene por objeto generalizar todas las cuestiones que se pueden proponer sobre las cantidades, el concepto Algebraico de cantidad es mucho más amplio que el aritmético.

Este trabajo de investigación presenta el diseño y aplicación de una unidad didáctica. En esta se encuentran diferentes estrategias de enseñanza-aprendizaje, con las cuales los estudiantes podrán superar las dificultades que presentan el contenido de Álgebra. La unidad didáctica desarrolla una secuencia de enseñanza que vincule aspectos numéricos, geométricos y Algebraicos.

Conocer el lenguaje Algebraico permitirá ofrecer la vinculación de la aritmética con el álgebra mediante la orientación de aspectos numéricos y contextuales hacia ideas Algebraicas. Permitiéndonos describir como el alumno procesa su conocimiento y las dificultades que enfrenta mediante la caracterización de estrategias de resolución de problemas. Con la ayuda de este trabajo, los alumnos adquirirán la habilidad de plantear problemas que reflejen situaciones cotidianas, así como estrategias para resolverlas, mediante la realización de las lecturas y tareas propuestas. Su función principal es establecer y estructurar un idioma que ayuda a generalizar las distintas operaciones que se desarrollen dentro de la aritmética, teniendo en cuenta que una expresión Algebraica es una cadena de representaciones perteneciente al

\footnotetext{
${ }^{1}$ Trabajo dirigido por el licenciado Jairo José Flores.
} 
lenguaje Algebraico, el cual puede contener variables, números, así como también operaciones aritméticas.

Por tanto es necesario saber enseñar y como enseñar es uno de nuestros principales objetivos al realizar este trabajo investigativo, como maestros de la asignatura sabemos que la comprensión de las expresiones Algebraicas es fundamental, y el hacer de esta una asignatura más amigable para nuestros estudiantes tratando de acercar académicamente el contenido estudiado con la realidad del mundo que nos rodea.

\section{MATERIALES Y MÉTODOS}

Según su diseño el estudio corresponde a la investigación acción la cual se realiza con problemas prácticos cotidianos experimentados por los profesores, en vez de "problemas teóricos" definidos por los investigadores puros en el entorno de la disciplina del saber. Según el período de tiempo, la investigación es transversal la que consiste en analizar ¿cuál es el nivel o estado de una o diversas variables en un momento dado? Desde un enfoque filosófico, la investigación es cualitativa que es un método de investigación usado principalmente en las ciencias sociales que se basa en cortes metodológicos basados en principios teóricos tales como la fenomenología, hermenéutica, la interacción social empleando métodos de recolección de datos que son no cuantitativos, con el propósito de explorar las relaciones sociales y describir la realidad tal como la experimentan los correspondientes.

El universo está comprendido por los estudiantes de octavo grado del instituto San Sebastián de Acoyapa, en el que existen tres secciones en el turno matutino, para un total de 142 estudiantes. De estos, 81 son niñas. Consideramos tomar como muestra una sección completa para que el estudio de investigación sea más representativo y veraz. Esta muestra es no probabilística, el principal criterio de la selección de la misma es el octavo A, el cual fue seleccionado de las tres secciones al azar. Este grupo está conformado por 47 estudiantes, 28 de ellos son niñas (entre los 12 a 17 años de edad) y 19 varones (entre las edades de 12 a 18 años).

Se utilizaron tres técnicas para soportar el trabajo de investigación:

La Entrevista: Esta técnica de investigación basada en un intercambio de información que se efectúa cara a cara, con la cual conversamos con los docentes del Instituto, acerca de las necesidades de los estudiantes, nos dimos cuenta la forma en que el docente preparaba su clase (plan diario, fichas de contenidos, etc.), nos expresaron las dificultades en el aprendizaje de los estudiantes (motivación, interés, problemas familiares, etc.), la falta de material didáctico (alumnos y docente) y sobre todo lograr una recolección de simpatía con el docente, lo cual fue fundamental en el transcurso de nuestra investigación.

La Observación: Se utilizó esta técnica para observar a los docentes en su labor, con la cual nos permitió determinar ¿qué está haciendo?, ¿qué hizo?, ¿cuánto tiempo toma?, ¿cómo lo distribuye?; Pero sobre todo darnos cuenta de las características de los estudiantes.

La Encuesta: Una vez seleccionada nuestra muestra (Alumnos de octavo "A") se procedió a la aplicación de la encuesta destinada específicamente para obtener información relevante de esta población de interés. Se decidió usar este tipo de técnica porque era la mejor manera de diagnosticar el grado de conocimiento de relacionar las matemáticas a situaciones del contexto; pero sobre todo la introducción a la nueva unidad como es el Álgebra.

\section{RESULTADOS}

Para enseñanza y aprendizaje del Álgebra es fundamental dominar el concepto de variable y en este caso de Expresiones Algebraicas. La posibilidad de representar en una sola letra un conjunto de valores y el 
hecho de poder manejarlos de forma sencilla es, precisamente lo que hace que el álgebra sea de gran utilidad, sin embargo los alumnos no llegan a comprender y aprovechar la ventaja que supone esta utilización de símbolos porque no llegan a ver su relación con lo que representan. Se observó que la utilización de letras les lleva a confusiones.

Al aplicar las actividades de la unidad didáctica, inicialmente, el estudiante se muestró temeroso al cambio, pero gradualmente fue aceptando y adaptándose como suele suceder en la vida. La primera actividad era un juego, con el que se pretendía desarrollar la capacidad de percibir aspectos generales, relaciones, cantidades y la relación de estas con el medio. En la actividad dos de la unidad didáctica, el estudiante puso en práctica esos conocimientos y utilizando letras y números escribía en forma Algebraica situaciones contextuales.

Ambas actividades fueron exitosas ya que el estudiante expresó que era algo innovador, diferente, que los mantuvo motivados desde el principio hasta el final y no la forma monótona la que estaban acostumbrados. Con la aplicación de todas y cada una de las actividades, los resultados obtenidos fueron los esperados. Se pretendía motivar tanto a los docentes como a los estudiantes sobre la importancia de un determinado tema y en este caso el de las Expresiones Algebraicas. Esto conducirá a elevar la calidad profesional de los docentes que imparten la disciplina de matemática en los centros públicos del país, fortaleciendo y afianzando las competencias didácticas.

El dominio de un tema por parte de los estudiantes es lo que todo maestro espera. Pero lograrlo como vimos no es una tarea fácil requiere de dedicar más de su tiempo y esfuerzo de su parte. Los resultados que se observan cuando un estudiante está motivado son satisfactorios, además que cuando ellos aprenden jugando es aún más fresco e innovador que es lo que pretendíamos lograr con nuestro trabajo.

\section{CONCLUSIONES}

Basados en la aplicación de los instrumentos, se reflejó el gran reto de la educación en relación a las matemáticas, siendo necesario el saber enseñar y ¿cómo hacerlo?

En los estudiantes, se notó una necesidad de actualización y de cambio. Esto les permitirá conocer e identificar las definiciones y propiedades de los objetos matemáticos, siendo capaces de usar el lenguaje Algebraica en la resolución de problemas. Por esto se puntualiza la necesidad de establecer puentes entre la matemática y la realidad natural y social que rodea a los jóvenes, basándose en aquello que los motive y que pueda despertar su interés logrando así un aprendizaje más significativo.

\section{BIBLIOGRAFÍA}

Cabezas M. (2000). El desarrollo psico-sexual del niño. Managua, Nicaragua: Fondo editorial CIRA.

Cabre, T. (1999) "La terminología, representación y Comunicación" IULA, Universitat Pompeu Fabra, Barcelona.

Cole, M. (2002) "Cultura, mente y actividad" Oxford, EE.UU.

Delors, J. (1997) "La educación encierra un tesoro. Informe de la Comisión Internacional sobre la Educación para el siglo XXI". Ediciones UNESCO, impreso en México.

González, O. (1996) "El enfoque histórico cultural como fundamentación de una concepción pedagógica", en Tendencias Pedagógicas Contemporáneas. Colombia: El Poiras Editores e Impresores, Ibagué.

Leontiev, A. N. (1981) “Actividad, conciencia, personalidad”. La Habana, Cuba; Pueblo y Educación. 